\title{
Panebianco, Stefano (2012): Standortfaktor Regional Governance auf dem Prüfstand. Theoretische Überlegungen und empirische Analysen zur Bedeutung regionaler Wirtschaftssysteme für die Wirtschaftsentwicklung von Regionen
}

\author{
Hamburg: Verlag Dr. Kovac. $=$ Schriftenreihe Wirtschaftspolitik in Forschung \\ und Praxis, Band 65. 508 S., 43 Abb., 88 Tab.
}

\section{Christian Diller}

Eingegangen: 30. August 2013 / Angenommen: 8. Oktober 2013 / Online publiziert: 23. Oktober 2013

(C) Springer-Verlag Berlin Heidelberg 2013

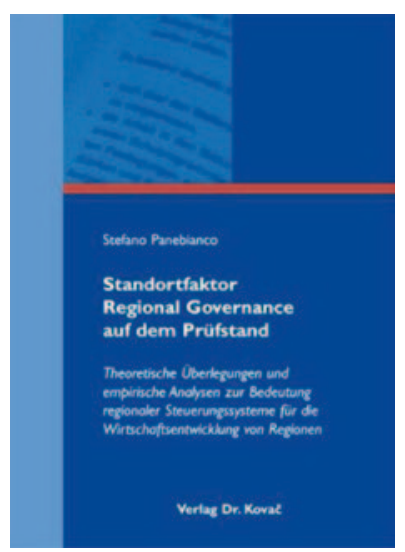

Die theoretische Debatte um den Begriff Regional Governance erreichte in Deutschland vor etwa zehn Jahren einen Höhepunkt; mit den Definitionen und Typisierungsvorschlägen von Benz/Fürst (2003) wurde eine Grundlage für weitere empirische Untersuchungen geschaffen. Gegenüber der normativen und in der Praxis häufig verwendeten Definition von Regional Governance, die vor allem die „weichen“, informellen netzwerkartigen Steuerungselemente in den Fokus nimmt, hat sich mittlerweile in der Raumplanungsforschung eher eine breitere Begriffsverwendung durchgesetzt, die - in Fortführung der Steuerungsdebatte der 1980er und 1990er Jahre - das Augenmerk auf das Zusammenspiel unterschiedlicher „harter“ wie „weicher“ Steuerungsfor-

Prof. Dr. C. Diller $(\bowtie)$

Institut für Geographie, Justus-Liebig-Universität Gießen,

Senckenbergstraße 1, 35390 Gießen, Deutschland

E-Mail: christian.diller@geogr.uni-giessen.de men legt (vgl. Mayntz 2005). Betrachtet man die Publikationslandschaft, so hat die Intensität der Debatte um den Begriff Regional Governance in den letzten Jahren zwar etwas abgenommen. Dennoch wurden einige interessante empirische Arbeiten vorgelegt, in räumlicher Hinsicht mit einem Akzent auf dem Thema Metropolitan Governance (z.B. Blatter/Knieling 2009). Bemerkenswert sind dabei vor allem Ansätze, Regional Governance auch modellierend in eine systematische zeitliche Perspektive zu setzen (von Löwis 2012). Hilfreich war es zudem, dass mit dem „Akteurzentrierten Institutionalismus“ eine Forschungsheuristik zunehmend Anwendung fand, die es ermöglicht, eine gewisse Vergleichbarkeit von empirischen Untersuchungen herzustellen (vgl. z. B. Lahner 2009).

Zwei Lücken können in der deutschen Regional Governance-Debatte dennoch festgestellt werden: Zum einen fällt auf, dass die grundlagenorientierte Forschung vor allem an den Entstehungsprozessen (z. B. kommunikative Konstruktion von Regional Governance, vgl. Kilper 2010) sowie den Anreizmechanismen (z. B. Wettbewerbe, vgl. Meincke 2012) und Funktionsweisen von Regional Governance interessiert war. Die Frage nach den Wirkungen von Regional Governance wurde jedoch an die Evaluationsforschung delegiert. Diese wiederum betrachtete, sofern sie sich nicht ohnehin ebenfalls nur auf die Governance-Strukturen und -Prozesse fokussierte, die Frage der Wirkungen nur auf der Mikroebene, indem beispielsweise direkte Outputs regionaler Kooperationen ermittelt wurden (z.B. Elbe 2007). Somit gibt es kaum empirische Erkenntnisse zu der Frage, wie sie welchen Einfluss auf das „Subsystem Regional Governance" bei der die Entwicklung des „Gesamtsystems Region" hat. 
Das zweite wesentliche Manko teilt Regional Governance mit weiten Teilen der deutschen Raumplanungsforschung: die fast schon phobisch zu nennende Vermeidung quantifizierender und generalisierender Ansätze. Es überwiegen bei Weitem Einzelfallbetrachtungen; bereits vergleichende Untersuchungen sind die Ausnahme, noch stärker generalisierende Versuche scheinen fast schon ein Tabu zu sein.

Vor diesem Hintergrund ist die Arbeit von Stefano Panebianco insofern bemerkenswert, als sie in Fragestellung und Methodik eher eine regionalökonomische, zum Teil regionalstatistische als eine planungs- oder politikwissenschaftliche Perspektive einnimmt. Dazu arbeitet Panebianco zunächst im Teil A die theoretischen Erklärungsansätze für regionales Wirtschaftswachstum, das Konzept der Regional Governance und die Wirkungsweise des Standortfaktors Regional Governance auf. Neuland wird dann im Teil B der empirischen Beobachtungen betreten: Es werden Kriterien für Merkmale zur Messung des Erfolges von Regional Governance abgeleitet und zu den entsprechenden Leitfragen der Stand der Forschung dargestellt: Sind regionale Steuerungsstrukturen erfolgversprechender? Welche regionalwirtschaftliche Relevanz haben Netzwerke? Partizipation - ein Beitrag zu mehr Beschäftigungswachstum? Das Handeln von Politik und Verwaltung - ein Erfolgsfaktor? Schließlich werden die empirischen Befunde gegenübergestellt und ein Fazit gezogen.

Die bemerkenswerte Leistung der Arbeit von Panebianco ist es, die verschiedenen Datenquellen zu einzelnen Indikatoren von Regional Governance für die Regionen in einer zeitlichen Perspektive flächendeckend für Deutschland auszuwerten. Es wurde ein Datenpool ausgewertet, der räumlich wie zeitlich deutlich auch über den Rahmen von Einzelevaluationen hinausreicht. Für Deutschland ist es vermutlich überhaupt die erste flächendeckende Untersuchung, die das Zusammenspiel „harter“ administrativer Verwaltungsstrukturen und „weicher" Issue-Networks betrachtet. Der wohl zentrale Befund der Untersuchungen: Während Wirkungen auf die Regionalentwicklung von durch Fördermittel unterstützten, netzwerkartigen regionalen Kooperationen, also der ,weichen Säule“ von Regional Governance, auch in einer langfristigen Perspektive nicht nachweisbar sind (anders als z. B. beim Einsatz von Mitteln aus der regionalen Wirtschaftsförderung, vgl. Bade 2012), korrelieren die Leistungsfähigkeit der kommunalen Verwaltungsstrukturen, also die ,harte Säule' von Regional Governance, und die regionale Wirtschaftsentwicklung positiv miteinander.

Dieses Ergebnis konsequent zu Ende zu denken (Panebianco selbst ist in der Interpretation eher zurückhaltend), hieße unter Umständen nach nunmehr über 20 Jahren ,,endogener Regionalentwicklung" die Ausrichtung und Struktur der gesamten entsprechenden Programmkulisse, vielleicht sogar ihre grundsätzliche Sinnhaftigkeit zu hinterfragen; denn: Einerseits schwindet die Basisfinanzierung von Krei- sen und Gemeinden, die für leistungsfähige und feste administrative Strukturen erforderlich ist. Andererseits werden die kurzfristig und teils kurzatmig angelegten Fördermittelanreize für zusätzliche Netzwerke immer weiter ausgebaut, was in einigen Regionen, wie Fürst (2003) schon vor Jahren einmal bemerkt hat, zu einem dauerhaften und sogar störenden Netzwerkrauschen führen kann.

Sicherlich: Die akribisch recherchierte, umfassende, aber dennoch gut lesbare Arbeit von Panebianco wird auch Kritiker finden. Man kann natürlich bezweifeln, ob es überhaupt angemessen ist, ,weiche' regionale Kooperation ,hart' zu evaluieren, und über die Aussagekraft einzelner Indikatoren lässt sich wie immer trefflich streiten. Manch einem mag das pragmatische Zusammensetzen einzelner theoretischer Erklärungsbausteine auf der einen und empirischer Daten auf der anderen Seite zu eklektizistisch erscheinen. Vermutlich aber genau durch dieses Vorgehen hat die stringente und konsistente Arbeit jedoch einen hohen praktischen Nutzen und öffnet auch für die Forschung neu Perspektiven zu dem Thema. Wenn durch sie die Barrieren gegenüber quantifizierender Forschung in der Raumplanung weiter abgebaut würden, so wäre dies schon ein sehr erfreulicher - und zum Teil ja sogar messbarer - Effekt.

\section{Literatur}

Bade, F.-J. (2012): Die Förderung gewerblicher Investitionen durch die Gemeinschaftsaufgabe „Verbesserung der regionalen Wirtschaftsstruktur": Wie erfolgreich sind die geförderten Betriebe? In: Raumforschung und Raumordnung 70 (1), 31-48.

Benz, A.; Fürst, D. (2003): Region, Regional Governance, Regionalentwicklung. In: Adamaschek, B.; Pröhl, M. (Hrsg.): Regionen erfolgreich steuern. Regional Governance - von der kommunalen zur regionalen Strategie. Gütersloh, 11-66.

Blatter, J.; Knieling, J. (2009): Metropolitan Governance - Institutionelle Strategien, Dilemmas und Variationsmöglichkeiten für die Steuerung von Metropolregionen. In: Knieling, J. (Hrsg.): Metropolregionen. Innovation, Wettbewerb, Handlungsfähigkeit. Hannover, 224-269. = Forschungs- und Sitzungsberichte der ARL 231.

Elbe, S. (2007): Die Voraussetzungen der erfolgreichen Steuerung integrierter Ansätze durch Förderprogramme: untersucht am Beispiel des Modellvorhabens Regionen Aktiv. Aachen.

Fürst, D. (2003): Aufwertung der Region als Steuerungsebene? In: Fürst, D.; Löb, S.; Rudolph, A.; Zimmermann, K. (Hrsg.): Steuerung durch Regionalplanung. Baden-Baden, 17-30.

Kilper, H. (2010): Governance und die soziale Konstruktion von Räumen. Eine Einführung. In: Kilper, H. (Hrsg.): Governance und Raum. Baden-Baden, 9-26.

Lahner, M. (2009): Regional Governance in Biosphärenreservaten. Eine Analyse am Beispiel der Regionen Rhön und Schaalsee unter Einbeziehung von Place Making. Stuttgart.

Mayntz, R. (2005): Governance Theory als fortentwickelte Steuerungstheorie? In: Schuppert, G.F. (Hrsg.): Governance-Forschung. Vergewisserung über Stand und Entwicklungslinien. Baden-Baden, 11-20.

Meincke, A. (2012): Regionen im Wettbewerb - Leistungswettbewerb als Steuerungsinstrument regionaler Netzwerke. Baden-Baden.

von Löwis, S. (2012): Governance in Stadtregionen zwischen Stabilität und Wandel. Entwicklung der räumlichen Steuerungsstrukturen in der Metropolregion Hamburg von 1989 bis 2009. Baden-Baden. 